\title{
Assessment of Postoperative Functional Outcome Following Brachial Plexus Palsy: Development and Validation of the Ioannina Functional Scale
}

\section{Magdalini S Stamou ${ }^{1 *}$, Marios D Vekris², Eleana N Bontioti $^{2}$ and Anastasios Korobilias ${ }^{2}$}

${ }^{1}$ Department of Physiotherapy, University of West Attica, Greece

${ }^{2}$ Department of Orthopedics, University Hospital of Ioannina, University of Ioannina

Medical School, Ioannina, Greece

*Corresponding Author: Magdalini S Stamou, Laboratory Teaching Stuff,

Physiotherapy Department, University of West Attica, Athens, Greece.
Received: April 27, 2020

Published: May 19, 2020

(c) All rights are reserved by Magdalini S

Stamou., et al.

\begin{abstract}
Aim: To create and validate a new assessment tool (Ioannina Functional Scale) for the postoperative evaluation of patients with Methods: The study included 57 patients who suffering lesion to the brachial plexus and had undergone surgery at the Orthopaedic Clinic at the Faculty of Medicine of the University of Ioannina. All procedures were led by the same surgeon (M.D.V.) The collection of data for the research was carried out by reviewing patients' files, interviewing patients and retrieving information from their physiotherapists. In all cases, written consent was obtained from the patients. Previous assessments tools for evaluating upper extremity function like the Disabilities of the Arm, Shoulder and Hand (D.A.S.H.), the Pain Visual Analogue Scale (P.V.A.S.) and the International Classification of Functioning, Disability and Health (I.C.F.) constituted the baseline of our comparison. Multiple statistical methods were used to establish the validity and reliability of the I.F.S. Pearson's and Spearman's tests were used for validation, Cronbach's alpha procedure for reliability, and Shapiro-Wilk for normality.

Results: Mean value of index for the I.F.S. was 49.81. A positive and very strong linear correlation between I.F.S. and D.A.S.H. indexes was documented. Distribution of the I.F.S. was found to be normal. Correlation between I.F.S. and P.V.A.S. were statistically significant with $\mathrm{p}=0.000$.

Conclusion: Based on the reliability and validity tests carried out in the present study, the Ioannina Functional Scale was found to be a useful assessment tool for evaluating the postoperative progress in patients with brachial plexus injury.

Keywords: Brachial Plexus Injury; Brachial Plexus Palsy; Clinical Assessment Tools; Clinimetrics
\end{abstract}

\section{Abbreviations}

I.F.S: Ioannina Functional Scale; D.A.S.H: Disabilities of the Arm, Shoulder and Hand; P.V.A.S.: Pain Visual Analogue Scale; I.C.F.: International Classification of Functioning, Disability and Health

\section{Introduction}

Brachial plexus palsy is associated with significant loss of upper extremity function in usually young and otherwise healthy patients. The functional impairment results in prolonged rehabilitation programs in the vast majority of patients and in many cases to permanent disability.

The postoperative course differs among patients with brachial plexus palsy depending on the severity of the paralysis, and the treatment methods. Adequate assessment of such patients is of crucial importance for both the rehabilitation team resulting in optimal improvement of upper extremity functionality and the surgeon in order to plan future operations that could further improve this functionality. Precise evaluation of patient's postoperative course is a useful tool in mapping of mobility and sensory possibilities that arise during the reanimation phase which allows for the readjustment and amendment of the therapeutic scheme. The recording and verification of the progress of rehabilitation programs through clinical scores increases the feedback between patient and treating clinicians and aids in monitoring patient's progress and provide documented information as to the effectiveness of current treatment.

Till recently, the assessment of patients with brachial plexus palsy targeted the mapping of mobility and sensibility of the upper extremity in relation to the degree of nerve regeneration. The assessment tools reported in the published literature are based on the evaluation of range of motion, muscular strength and sensory changes [1-3]. However, during the last few years an increasing need for evaluation of further parameters such as functionality and patients' quality of life has arisen [3-5]. 


\section{Aim of the Study}

The aim of this study is the creation of a simple, easily applicable and quick-to-use assessment tool for the evaluation of patients with brachial plexus palsy that can be used to map and link the postoperative clinical picture of patients' mobility, sensibility and functionality. The Ioannina Functional Scale (I.F.S.) was designed in order to record the postoperative muscular and sensory functionality of patients with brachial plexus palsy in order to assess the course of the rehabilitation program.

\section{Materials and Methods}

Patients

Fifty seven patients who had suffered a global brachial plexus lesion were initially considered eligible to participate in the present study. In all cases, written informed consent was obtained from the patients. From the 57 patients initially included in the study, 17 patients were excluded due to lack of compliance to the physiotherapy program. Eventually, 40 patients participated in the present study.

The mean follow up time was 3.35 years $(+/-1.1)$. The median age of patients at the time of operation was 23 years (range 15 - 35 years). The vast majority of the patients were males (36 patients). The denervation period was 1 - 3 months in 27.5\%, 4 - 6 months in $57.5 \%$ and more than 6 months in $15 \%$ of our sample population In $72.5 \%$ of the sample, avulsion of nerve root(s) was present (3 patients with complete avulsion of allnerve roots, 10 with avulsion of four roots, 8 with avulsion of three roots, 6 with avulsion of two roots and 2 patients with avulsion of one nerve root).

\section{Surgical technique}

All patients were operated at the Department of Orthopaedic Surgery of the University Hospital of Ioannina. All procedures were performed by the same surgeon (M.D.V.). Exploration of the brachial plexus was performed and depending on whether the donor nerve was part of the brachial plexus or not, the neurotisation was categorised as intraplexus or extraplexus, where:

- Intraplexus neurotisation is neurotisation using donor nerves harvested from within the brachial plexus (C5 and C6 roots).

- Extraplexus neurotisation is neurotisation using donor nerves harvested from outside the brachial plexus (the spinal accessory nerve, the phrenic nerve, the cervical plexus motor donor, the intercostal nerves and the contralateral C7 root).

Neurotisation of the suprascapular nerve was performed in all patients since it is considered as one of the primary targets in bra- chial plexus reconstruction along with musculocutaneous nerve. Therefore, both these nerves were reconstructed in all patients. For the suprascapular nerve reconstruction, the spinal accessory nerve was used as donor nerve in $80 \%$ of patients, the $\mathrm{C} 5$ root was used in $10 \%$, the branch of the cervical plexus was used in $5 \%$ and the phrenic nerve was used in the remaining $5 \%$. Neurotisation of the axillary nerve was carried out in $60 \%$ of the patients. The C5 root was used as donor in $65 \%$, the phrenic nerve in $12.5 \%$ and a branch of the cervical plexus was used in $17.5 \%$. For elbow flexion, neurotisation of the musculocutaneous nerve was performed in all patients included in the study. The phrenic nerve was used in $45 \%$, branches of the $\mathrm{C} 5$ root in $32 \%$, the ulnar nerve in $15 \%$ and the spinal accessory nerve in $8 \%$. Neurotisation of the median nerve was carried out in $70 \%$ of our sample. C5 root was used in $60 \%$, and intercostal nerves was used as donor in $40 \%$. Generally, intraplexus neurotisation was used in $32.5 \%$ of our patients and extraplexus neurotisation in $77.5 \%$.

\section{Postoperative assessment}

At 6 weeks postoperatively, the patients started physiotherapy and electrotherapy protocol. All patients were evaluated at 4 month intervals till the final evaluation that was performed at 24 months postoperatively. The evaluation of patients was performed with a novel functional scale, introduced by our institution (Ioannina Functional Scale). Moreover, all patients were also evaluated with the International Classification of Functioning, Disability and Health (ICF) the Disabilities of the Arm, Shoulder and Hand (DASH) [6] and the Pain Visual Analogue Scale (PVAS) [7] assessment tools in order to test the credibility and external validity of the Ioannina Functional Scale. All assessment tools were used in Greek language.

Description of the Ioannina Functional Scale (I.F.S.)

The I.F.S. is divided into four sections describing:

- A: joint's range of motion,

- B: muscular strength,

- C: functionality and

- D: protective sensibility.

The two first sections (A and B) record the mobility and muscular strength of the injured structures and describe the course of rehabilitation. More precisely:

- A: Section for the recording of range of motion of the upper extremity. Section A is divided into subsections:

- A1: Recording the passive range of motion.

An extremity's passive range of motion provides us with information about the condition of the joint structures after the 
Functional assessment scale for brachial plexus injuries

$A$ : Range of motion of the upper extremity

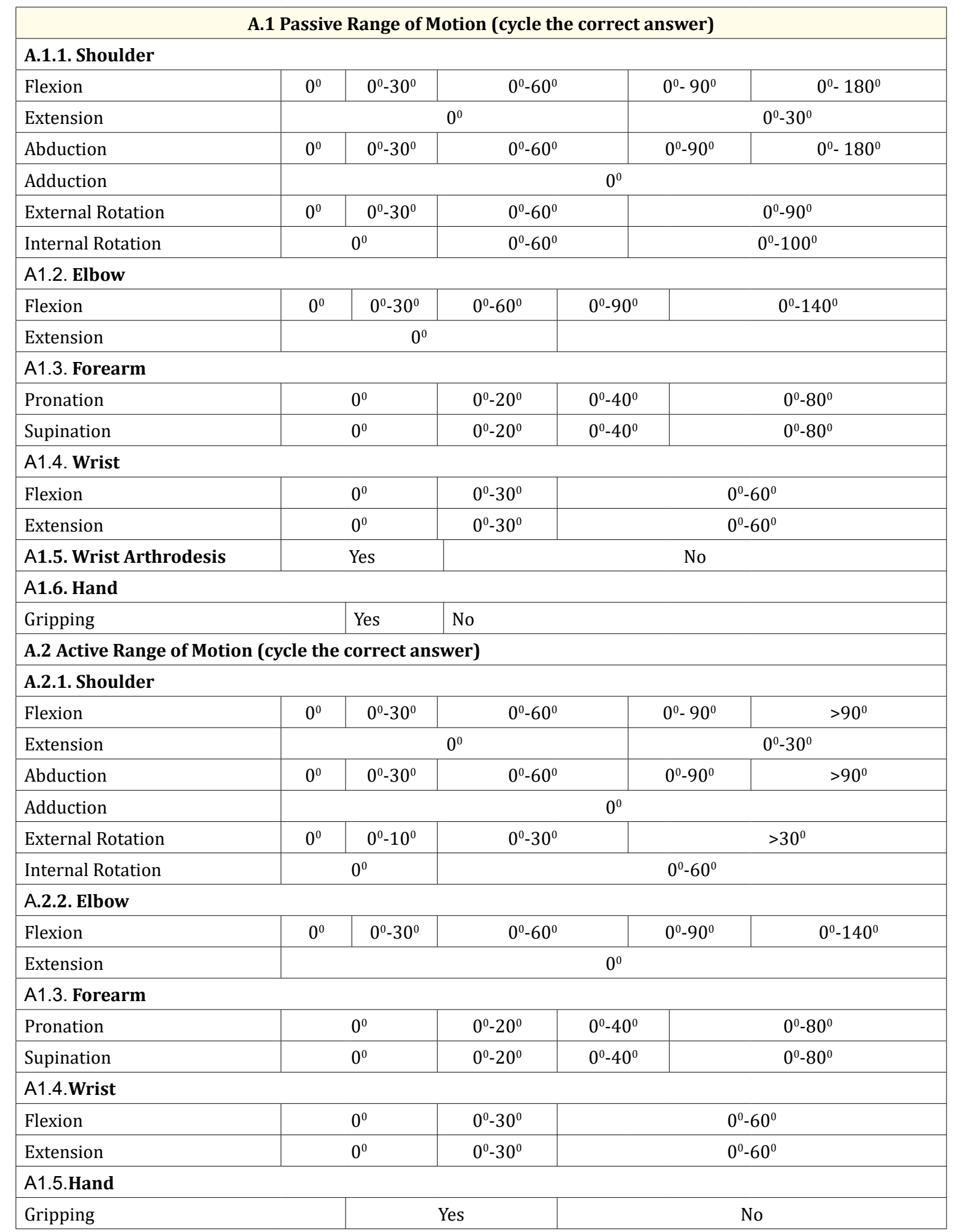

\begin{tabular}{|c|c|}
\hline B. Muscle strength & B.M.R.C Grading System Expanded with (+) and (-) \\
\hline \multicolumn{2}{|l|}{ B.1 Shoulder } \\
\hline \multicolumn{2}{|l|}{ Muscle Groups for Shoulder Girdle Elevation } \\
\hline Rhomboids & \\
\hline Trapezius & \\
\hline
\end{tabular}




\begin{tabular}{|c|c|c|}
\hline \multicolumn{2}{|c|}{ Muscle Groups for Shoulder Abduction } & \\
\hline & Deltoid & \\
\hline & Supraspinatus & \\
\hline & Serratus Anterior & \\
\hline \multicolumn{2}{|c|}{ Muscle Groups for Shoulder External Rotation } & \\
\hline & Infraspinatous & \\
\hline & Teres Minor & \\
\hline \multicolumn{2}{|c|}{ Muscle Groups for Shoulder Flexion } & \\
\hline & Anterior Deltoid & \\
\hline & Pectoralis major & \\
\hline \multicolumn{2}{|c|}{ Muscle Groups for Adduction and Internal Rotation } & \\
\hline & Latissimus Dorsi & \\
\hline & Pectoralis major & \\
\hline \multicolumn{2}{|l|}{ B2. Elbow } & \\
\hline \multicolumn{2}{|c|}{ Muscle Groups for Elbow Flexion } & \\
\hline B2.1 & Bicep & \\
\hline B2.2 & Brachioradialis & \\
\hline \multicolumn{2}{|c|}{ Muscle Groups for Elbow Extension } & \\
\hline B2.3 & Triceps & \\
\hline \multicolumn{2}{|l|}{ B.3 Wrist } & \\
\hline B3.1. & Wrist Extensors (E.C. Radialis B./L. -E.C. Ulnaris) & \\
\hline B3.2 & Pronator & \\
\hline \multicolumn{3}{|l|}{ B.4 Hand } \\
\hline B4.1 & Fingers Flexion & \\
\hline B4.2 & Fingers Extension & \\
\hline
\end{tabular}

\begin{tabular}{|c|c|c|c|c|c|c|}
\hline \multicolumn{2}{|r|}{ C. Ability to Execute Everyday Activities. } & $\begin{array}{c}\text { No } \\
\text { Difficulty } \\
\end{array}$ & $\begin{array}{c}\text { Mild } \\
\text { Difficulty }\end{array}$ & $\begin{array}{l}\text { Moderate } \\
\text { Difficulty }\end{array}$ & $\begin{array}{c}\text { Severe } \\
\text { Difficulty }\end{array}$ & Unable \\
\hline C1 & Lifting large object (such as a bag) & 0 & 1 & 2 & 3 & 4 \\
\hline $\mathrm{C} 2$ & Carrying large object (such as a bag or a chair) & 0 & 1 & 2 & 3 & 4 \\
\hline C3 & Open a door & 0 & 1 & 2 & 3 & 4 \\
\hline $\mathrm{C} 4$ & Close a door & 0 & 1 & 2 & 3 & 4 \\
\hline $\mathrm{C} 5$ & Picking up small objects & 0 & 1 & 2 & 3 & 4 \\
\hline C6 & Push small objects & 0 & 1 & 2 & 3 & 4 \\
\hline C7 & Table/office cleanness in a circular motion & 0 & 1 & 2 & 3 & 4 \\
\hline \multicolumn{2}{|r|}{ D. Protective Sensibility and Pain } & $\begin{array}{c}\text { No } \\
\text { Difficulty } \\
\end{array}$ & $\begin{array}{c}\text { Mild } \\
\text { Difficulty }\end{array}$ & $\begin{array}{l}\text { Moderate } \\
\text { Difficulty }\end{array}$ & $\begin{array}{c}\text { Severe } \\
\text { Difficulty }\end{array}$ & Unable \\
\hline D1 & Sensory function of movement & 0 & 1 & 2 & 3 & 4 \\
\hline D2 & Sensory functions of sensing cold and heat & 0 & 1 & 2 & 3 & 4 \\
\hline D3 & Sensitivity to a noxious stimulus & 0 & 1 & 2 & 3 & 4 \\
\hline D4 & Pain & 0 & 1 & 2 & 3 & 4 \\
\hline
\end{tabular}

Table 1: Ioannina functional scale. 
immobilisation or the possible restrictions that might occur due to fractures or scar tissue from the initial trauma.

- A2: Recording the active range of motion.

An extremity's active range of motion shows the degree of functionality that can be achieved.

- B. Section for the recording of muscular strength of the upper extremity with the British Medical Research Council (BMRC) Grading System [8] expanded further with intermediate grades of (+) and (-) (i.e. M2, M2+, M3-, M3, M3+) [9,10].

The measuring of muscular strength of every affected muscle separately, gives us a specific clinical picture of each individual patient.

- C. Section for the recording of ability to execute everyday activities.

Refers to a list of basic activities involving the injured limb i.e. carrying of a bag or a chair. The degree of difficulty in executing these tasks is recorded.

- D. Section for the recording of protective sensibility and pain.

Recording in a chart of protective sensibility such as the feeling of hot/cold, proprioception and pain, as subjectively perceived by the patient.

\section{Statistical procedures}

The DASH, ISF and IFS index were calculated. The IFS index was calculated with the same mathematical type as the DASH in$\operatorname{dex}$ (Dash Disability Symptom Score $=[$ (sum of $\mathrm{n}$ responses) -1$] \mathrm{x}$ 25 , where $n$ is equal to the number of completed responses). Were coded the categories of the ten variables, transformed them into a 100-grade scale and then calculated the I.F.S. The index values nearing 100 correspond to complete difficulty while the values nearing 0 constitute no difficulty at all. The new functional scale was evaluated for its internal and external validity.

In order to evaluate the normal distribution of our sample for the DASH and IFS questionnaires, we used the KolmogorovSmirnov and Shapiro-Wilk tests. The Shapiro-Wilk test is recommended for small samples, in which values greater or lesser than 1.96 are sufficient to establish normality of the data.

To check the reliability of IFS and DASH questionnaires, we used the Cronbach's alpha coefficient for internal consistency. For this procedure, values of between 0.70 and 0.90 are considered satisfactory.
For the external validation of the IFS, we correlated the IFS scale with the DASH, the PVAS and the ICF using the Pearson's and Spearman's correlations. P values $<0.05$ were considered statistically significant; all $p$ values were two-tailed. All statistical analyses were performed using the Statistical Package for Social Sciences (SPSS 22.0, Chicago, IL, USA)

\section{Results and Discussion}

The disability index of the D.A.S.H. ranged from 8.33 to 94.17 with the mean value being $56.25+/-24.12$, which corresponds to a medium level of disability in the sample population. The disability index of the I.F.S. ranged from 5 to 97.50 and the mean value of the index was $49.81+/-27.24$. This also corresponds to a medium disability level in the sample population in accordance to the D.A.S.H. score. The distribution of the I.F.S. and D.A.S.H. indexes respectively is shown in figure 1. The mean value of P.V.A.S. was $4.20+/-2.75$.

In order to examine the normality of our sample population for the D.A.S.H. questionnaire, we used the Kolmogorov-Smirnov and

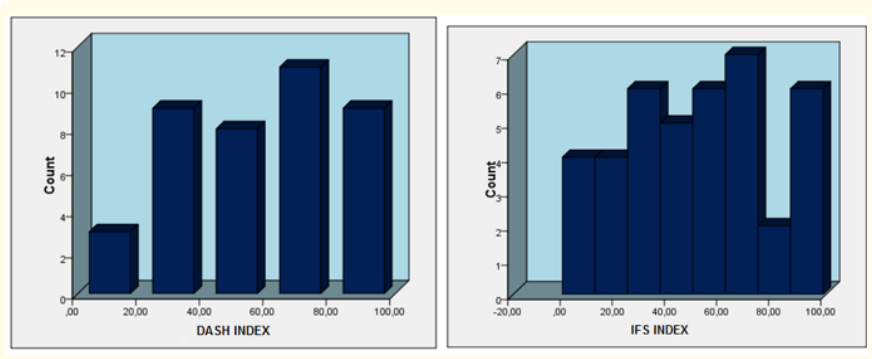

Figure 1

Shapiro-Wilk tests. We noted that Sign. $=0.20$ for the KolmogorovSmirnov test and Sign. $=0.196$ for the Shapiro-Wilk test. Both $\mathrm{p}$ values were $<0.05$, which shows statistical significance indicating a normal sample distribution. In concordance to the previous results, a normal sample distribution was also seen when the I.F.S. questionnaire was used (Sign. $=0.20$ with the Kolmogorov-Smirnov test and Sign. $=0.176$ withthe Shapiro-Wilk test).

The estimation of the reliability of the I.F.S. was checked with the coefficient of the internal consistency Cronbach's alpha. The alpha coefficient for all participants $(\mathrm{N}=40)$ for all ten questions in the I.F.S. is 0.964 , suggesting that the items have relatively high internal consistency.

\section{D.A.S.H. index and P.V.A.S.}

As expected, a strongly positive and statistically significant correlation was noted between the DASH and PVAS scores with $\mathrm{p}<$ 0.001 . 


\begin{tabular}{|c|c|c|c|c|c|}
\hline \multicolumn{2}{|r|}{ I.F.S. Variable } & \multicolumn{2}{|c|}{ I.C.F./ D.A.S.H. Variables } & \multirow{2}{*}{$\begin{array}{c}\begin{array}{c}\text { Correlation Coefficient } \\
\text { Spearman }\end{array} \\
0,950\end{array}$} & \multirow{2}{*}{$\begin{array}{r}\begin{array}{r}\text { Statistically } \\
\text { Significant }\end{array} \\
\sqrt{ }\end{array}$} \\
\hline I.F.S. - C1 & $\begin{array}{l}\text { Lifting large object } \\
\text { (such as a bag) }\end{array}$ & I.C.F._d430 & Lifting and carrying object & & \\
\hline I.F.S. - C2 & $\begin{array}{l}\text { Carrying large object } \\
\text { (such as a bag or a chair) }\end{array}$ & I.C.F._d4302 & Carrying in the arms & 0,963 & $\sqrt{ }$ \\
\hline I.F.S. - D1 & Sensory function of movement & I.C.F._b260 & $\begin{array}{l}\text { Proprioceptive sensory } \\
\text { function }\end{array}$ & 0.966 & $\sqrt{ }$ \\
\hline I.F.S. - D2 & $\begin{array}{l}\text { Sensory functions of sensing } \\
\text { cold and heat }\end{array}$ & I.C.F._b2700 & $\begin{array}{l}\text { Sensory functions of } \\
\text { sensing cold and heat }\end{array}$ & 0.966 & $\sqrt{ }$ \\
\hline I.F.S. - D3 & $\begin{array}{l}\text { Sensitivity to a noxious } \\
\text { stimulus }\end{array}$ & I.C.F._b2703 & $\begin{array}{l}\text { Sensory functions of sens- } \\
\text { ing painful or } \\
\text { uncomfortable } \\
\text { sensations }\end{array}$ & 0.966 & $\sqrt{ }$ \\
\hline I.F.S. - D4 & Pain & I.C.F._b280 & Sensation of pain & 0.978 & $\sqrt{ }$ \\
\hline I.F.S. - C3 & Open a door & D.A.S.H.5 & Push open a heavy door & 0,941 & $\sqrt{ }$ \\
\hline I.F.S. - D4 & Pain & D.A.S.H.24 & $\begin{array}{l}\text { Arm, shoulder or hand } \\
\text { pain. }\end{array}$ & 0.825 & $\sqrt{ }$ \\
\hline I.F.S. - D4 & Pain & VAS & Pain Scale & 0.912 & $\sqrt{ }$ \\
\hline
\end{tabular}

Table 2

External validity - Correlation of the I.F.S. index with other scores

Using the Spearman's correlation coefficient, a strongly positive and statistically significant correlation was seen between the IFS and the PVAS, with $\mathrm{p}$ values $<0.001$ (Table 2 ). In addition, a positive and very strong linear correlation between the I.F.S. index and the D.A.S.H. index was seen $(\mathrm{p}<0.001)$ (Table 2). All comparisons carried out between the variables of the I.F.S. and relevant variables of the I.C.F. exhibited a statistically significant correlation (Table 2).

The above results led us to the conclusion that the I.F.S. index is equally capable of indicating the functional disability while making use of fewer questions than the D.A.S.H. for the patients in our sample.

\section{Conclusion}

The assessment of the postoperative course in patients with brachial plexus palsy is of crucial importance by evaluating the sensory motor improvement during the reinnervation phase. More- over, the assessment of functionality provides valuable information for the readjustment and amendment of the therapeutic protocol, the motivation and feedback to patient and therapist.In general, patients with injury to the brachial plexus face loss of active mobility, weakness of the upper limb and altered sensibility [11]. In this particular group of patients, rehabilitation is considered a success when the patient regains "useful" functionality. "Useful" rehabilitation is defined as partial motor control of the shoulder, strong elbow flexion and partial motor control of the hand. Millesi notes that $72 \%$ of patients regain "useful" functionality following neurolysis and 70\% following nerve grafting [12] Chuang DCC (2010) reports that successful rehabilitation of patients following nerve grafting is considered to be achieved when the patient exhibits a 180 degree shoulder flexion, M4 muscle strength, M4 to M4+ elbow flexion and extension, M3- to M3+ finger flexion and extension and M2 (or less) hand intrinsic muscles movement [13]. In the case of avulsion of nerve roots and where neurotisation has been performed, successful rehabilitation is considered to be achieved when the patient exhibits a 60 degree shoulder abduction, M4 elbow flexion and M2- 
M4 finger flexion. Extension of elbow and fingers can be achieved at a later stage with a free functional muscle transplant.

Knowing that the harmonic movement of the upper limb is no longer present following injury, the need for an assessment tool designed specifically to map the functional impairment of this particular patient group, is considered necessary. Till now, the assessment of postoperative course of patients with brachial plexus palsy, was based on tools such as the P.V.A.S and the D.A.S.H [12,1416]. Moreover, the I.C.F. is a useful framework for the classification of the effect on an individual after brachial plexus injury, where the impact of the injury on somatic structures and their function is classified according to the resulting restrictions and activity of the patient $[12,17]$. These tools are internationally recognised for their validity and when combined they can evaluate pain levels and functional rehabilitation of these patients.

However, the inflexible condition under which the D.A.S.H. can be used to assess the disability index, i.e. the answering of at least 27 of the 30 questions necessitated the development of a new assessment tool. In particular, during the patient interviews that we carried out, it became apparent that there are more than only three questions that cannot be answered by this particular group of patients in the D.A.S.H. questionnaire. There are also questions of which we know beforehand cannot be answered due to the inability of these patients to carry out these tasks. In general, at least seven questions in the D.A.S.H. questionnaire cannot be answered by patients with brachial plexus palsy, thus more than the three allowed for in the design of the D.A.S.H.

Being more specific ,questions like 'Open a tight or new jar", "Write" and "Turn a key" all assume a harmonic movement of the hand(supination/pronation - extension of fingers and thumb) and tasks such as "Change a light bulb overhead", "Wash or blow dry your hair", "Wash your back" and "Put on a pullover sweater" assume an ability to flex or abduct the shoulder more than 120 degrees, excellent flexion of the elbow and the concurrent harmonic movement of the wrist using supination and pronation. These are all movements which patients in postoperative useful rehabilitation of the brachial plexus cannot carry out. Since they are obliged to answer all but three, the index would not be representing the actual 'useful' recovery of patients.

Moreover, the psychological pressure on the patient, caused by asking questions that cannot reasonably be answered, is potentially enormous. Depending on personality and expectations for patient recovery, the perceived extent of loss as charted, could trigger and increase the emotional trauma of the initial injury and furthermore might interfere with their discipline or willingness to continue rehabilitation. The whole process of rehabilitation may seem pointless.
On the other hand, although variables of the I.C.F. relate to the weaknesses experienced by patients in the postoperative progress, i.e. lack of active movement, weakness of the upper extremity and changes in sensibility, the tool is descriptive and has not been designed to measure degree of disability.

All the above concerns necessitated the development of a new assessment tool, The Ioannina Functional Score. Our score showed a positive and very strong linear correlation with the D.A.S.H. index, indicating equal ability in mapping the functional disability of patients with brachial plexus palsy, while using fewer questions than the D.A.S.H. Moreover, in our study we used the relevant variables of the I.C.F. to assess the I.F.S. All comparisons carried out between the variables of the I.F.S. and relevant variables of the I.C.F. exhibited a statistically significant correlation. The advantage of I.F.S. over I.C.F. is the possibility to obtain an index [18-20].

In conclusion, based on the statistical analyses carried out in the present study, the Ioannina Functional Scale is a suitable tool for assessment of postoperative progress in patients with brachial plexus injury. It is simple, reliable and applicable to all brachial plexus patients. It correlates functional outcome with objective clinical measurements i.e. muscle strength and ROM and it provides clinicians with a comparable index.

\section{Bibliography}

1. Mackinnon SE, et al "Results of reinnervation of the biceps and brachialis muscles with a double fascicular transfer for elbow flexion". The Journal of Hand Surgery 30.5 (2005): 978-985.

2. Zimmerman NB., et al. "Are standardized patient self-reporting instruments applicable to the evaluation of ulnar neuropathy at the elbow?". Journal of Shoulder and Elbow Surgery 18.3 (2009): 463-468.

3. Bertelli JA and Ghizoni MF. "Brachial plexus avulsion injury repairs with nerve transfers and nerve grafts directly implanted into the spinal cord yield partial recovery of shoulder and elbow movements". Neurosurgery 52.6 (2003): 1389-1390.

4. Kitajima I., et al. "Evaluation of quality of life in brachial plexus injury patients after reconstructive surgery". Hand Surgery 11.3 (2006): 103-107.

5. Novak CB., et al. "Validity of the Patient Specific Functional Scale in patients following upper extremity nerve injury". Hand (N Y) 8.2 (2013): 132-138. 
6. Novak CB., et al. "Patient-reported outcome after peripheral nerve injury". The Journal of Hand Surgery American 34.2 (2009): 281-287.

7. Themistocleous GS., et al. "Translation into Greek, cross-cultural adaptation and validation of the Disabilities of the Arm, Shoulder, and Hand Questionnaire (DASH)". Journal of Hand Therapy 19.3 (2006): 350-357.

8. Huskisson EC. "Measurement of Pain". The Lancet 2.7889 (1974): 1127-1131.

9. Medical Research Council. "Aids to examination of the peripheral nervous system". Memorandum London: Her Majesty's Stationary Office 45 (1976).

10. Terzis JK., et al. "Outcomes of brachial plexus reconstruction in 204 patients with devastating paralysis". Plastic and Reconstructive Surgery 104.5 (1999): 1221-1240.

11. Vekris MD., et al. "Restoration of elbow function in severe brachial plexus paralysis via muscle transfers". Injury 39.3 (2008): 15-22

12. Hill BE., et al " Clinimetric Evaluation of Questionnaires Used to Assess Activity After Traumatic Brachial Plexus Lesion in Adults: A Systematic Review". Archives of Physical Medicine and Rehabilitation 92.12 (2011): 2082-2089.

13. Millesi H., et al. "Nomenclature in peripheral nerve surgery. Committee report of the International Society of Reconstructive Microsurgery". Clinics in Plastic Surgery 11.1 (1984): 3-8.

14. Chuang DCC. "Brachial Plexus Lesion: Nerve Reconstruction and Functioning Muscle Transplantation Extremity-Saving Surgery and Reconstruction: From Microsurgery Reconstruction to Transplantation". Seminars in Plastic Surgery 24.1 (2010): 57-66

15. Kretschmer T., et al. "Patient satisfaction and disability after brachial plexus surgery". Neurosurgery 65.4 (2009): 189-196.

16. Dodakundi C., et al. "Outcome of surgical reconstruction after traumatic total brachial plexus palsy". Journal of Bone and Joint Surgery American 95.16 (2013): 1505-1512.

17. Stucki G., et al. "Value and application of the I.C.F. in rehabilitation medicine". Disability and Rehabilitation 24.17 (2003): 932-938.

18. Smania N., et al. "Rehabilitation of brachial plexus injuries in adults and children". European Journal of Physical and Rehabilitation Medicine 48.3 (2012): 483-506.
19. Scott KR., et al. "Rehabilitation of brachial plexus and peripheral nerve disorders". The Handbook of Clinical Neurology 110 (2013): 499-514.

20. Land C., et al. "The Development and Evolution of Research on Social Indicators and Quality of Life (QOL)". Handbook of Social Indicators and Quality of Life Research (2012): 1-22.

\section{Assets from publication with us}

- Prompt Acknowledgement after receiving the article

- Thorough Double blinded peer review

- Rapid Publication

- Issue of Publication Certificate

- High visibility of your Published work

Website: https://www.actascientific.com/

Submit Article: https://www.actascientific.com/submission.php Email us: editor@actascientific.com

Contact us: +919182824667 